\section{Marking diabetes}

\section{By Joanne Kotz, Senior Editor}

A Massachusetts team has identified amino acid ${ }^{1}$ and triacylglyceride ${ }^{2}$ signatures that can predict increased risk of developing type 2 diabetes. The researchers now plan to look at whether the signatures can distinguish which prediabetic patients will benefit from lifestyle changes or therapeutic intervention. The key unknown is whether a cutoff value for the signatures can be identified that will prospectively predict an individual's risk or response to treatment.

"We know that the current tools for diagnosing diabetes-glucose and $\mathrm{HbAlc}$ - are not particularly good at predicting diabetes," said Steven Watkins, CSO of Tethys Bioscience Inc. "This is because changes in the blood levels of these markers tend to occur late in the disease process."

Tethys markets PreDx DRS, an assay that predicts an individual's risk of developing diabetes. The test is conducted in a CLIA-certified laboratory and incorporates seven circulating biomarkers including glucose and hemoglobin A1c (HbA1c).

In a search for other early markers of type 2 diabetes, a team led by researchers from Massachusetts General Hospital and the Broad Institute of MIT and Harvard conducted two studies. One looked at levels of metabolites, and the other looked at levels of lipids in the blood of 378 participants in the Framingham Offspring Study.

At the time of an examination conducted from 1991-1995, none of the participants had diabetes but all were judged to be at high risk for the disease. Out of this cohort, only half went on to develop diabetes over the next 12 years.

The Massachusetts team set out to identify the markers that could help better predict who would go on to develop the disease.

In serum samples from the 1991-1995 examinations, the team used mass spectrometry to identify metabolites or lipids that were present in different concentrations in the blood of people who went on to develop diabetes compared with in the blood of those who did not.

In the first study, three amino acids-isoleucine, tyrosine and phenylalanine-were present at higher levels in the serum of people who later developed diabetes. In the study analyzing lipid levels, triacylglycerides (TAGs) with shorter chain length and fewer double bonds were present at higher levels in the blood of people who later developed diabetes than TAGs with longer chain length or more double bonds.

After controlling for age, sex, BMI and fasting glucose, the top quartile of patients with the highest amino acid signature had a sixfold greater risk of developing diabetes than those having a signature in the bottom quartile.

Similarly, top-quartile TAG signature patients had a 4.3 -fold higher risk of becoming diabetic than bottom-quartile TAG signature patients.

Results from the metabolite study were published in Nature Medicine, and results from the lipid study were published in The Journal of Clinical Investigation.

"A relationship between branched-chain amino acids or triacylglyceride composition and diabetic processes has been known for a while, but this is the first large-scale clinical validation of the observation," said Watkins.

\section{Risk prediction}

The predictive power of these signatures for diabetes risk is real but relatively modest, said Watkins. Thus, he thinks the markers likely will need to be combined with other measures to provide clinically useful predictive power.

"These papers report intriguing markers," said Michael Milburn, CSO of Metabolon Inc. "Determining how practical they are is really the next step."

Metabolon is developing a test for insulin resistance that measures three metabolites and can be used to predict the risk of developing diabetes and other diseases. Milburn said the company hopes to launch the test around year end.

Because the current studies normalized for glucose levels, they did not directly test how these signatures compared to the predictive power of glucose levels, noted Milburn. Thus, he said it will be important to conduct additional studies to see how these signatures perform compared with current clinical standards such as glucose measurements.

In addition, Milburn thinks there will be challenges in moving from looking at a whole population to demonstrating clinical utility for a particular individual.

This is a key question to be addressed, agreed Christopher Newgard, professor of pharmacology and cancer biology and director of the Sarah W. Stedman Nutrition and Metabolism Center at the Duke University School of Medicine. "For a person off the street, what would be a cutoff level that constitutes a danger sign?" he asked.

Robert Gerszten and Thomas Wang, who co-led both studies, said the Massachusetts team now plans to run additional studies in larger, heterogeneous populations to examine how their findings can be generalized to individuals with a variety of backgrounds, for instance, different ethnicities and age groups.

Gerszten is director of the clinical and translational research program at the MGH heart center, associate professor of medicine at Harvard Medical School and a senior associate at the Broad Institute. Wang is associate director of the heart failure and transplantation program at $\mathrm{MGH}$ and associate professor of medicine at Harvard Medical School. 
Another angle being pursued by the team is to determine whether the amino acid and TAG signatures are independent of one another and whether they could be combined to predict diabetes risk more accurately. "The early look is that the signatures are not highly correlated, so they may well add information to each other, but this still needs to be proven formally," said Gerszten.

\section{Therapy guide}

Markers that more accurately predict the risk of an individual developing diabetes would clearly be useful clinical tools, but the real home run would be markers that can guide the individual treatment of prediabetic patients.

Prediabetes encompasses multiple different metabolic dysfunctions, including insulin resistance, $\beta$ cell dysfunction and dyslipidemia. These different conditions are "poorly differentiated with current tests," said Milburn, and an important future direction is identifying markers for each of these distinct conditions that can help predict response to different types of treatments.

There are some hints that the amino acid signature may be up to that task.

Newgard cited a 2010 study that looked mechanistically at the insulinsensitizing action of thiazolidinediones. ${ }^{3}$ In obese, insulin-resistant rats, the extent of tissue-specific transcriptional changes in the branched-chain amino acid pathway correlated with efficacy of four thiazolidinediones, including Avandia rosiglitazone from GlaxoSmithKline plc and Actos pioglitazone from Takeda Pharmaceutical Co. Ltd.

"Where we now need to go is to overlay pharmacotherapies that are available today on top of our understanding of the predictive nature of these amino acid signatures," said Newgard.

Watkins agreed that a next step will be determining whether interventions that change the marker concentrations lead to positive outcomes in individual patients.

Indeed, Gerszten said the Massachusetts team is planning to look at whether the amino acid or TAG signature can predict response to intervention. The team plans to look at drug and lifestyle intervention studies for prediabetics to see if either signature correlates with response.

Gerszten told SciBX that the team would be "interested in partnering with pharma to identify patient subsets that are most at risk for disease and/or most likely to derive benefit from therapeutic interventions."

Patent applications have been filed covering both diagnostic and therapeutic applications for the results reported in both papers and are available for licensing, said Gerszten.

Kotz, J. SciBX 4(16); doi:10.1038/scibx.2011.444

Published online April 21, 2011

\section{REFERENCES}

1. Wang, T.J. et al. Nat. Med.; published online March 20, 2011; doi:10.1038/nm.2307

Contact: Robert E. Gerszten, Massachusetts General Hospital and Harvard Medical School, Charlestown, Mass.

e-mail: rgerszten@partners.org

Contact: Thomas J. Wang, same affiliation as above e-mail: tjwang@partners.org

2. Rhee, E.P. et al. J. Clin. Invest.; published online March 14, 2011; doi:10.1172/JCl44442

Contact: Robert E. Gerszten, Massachusetts General Hospital and Harvard Medical School, Charlestown, Mass.

e-mail: rgerszten@partners.org

Contact: Thomas J. Wang, same affiliation as above e-mail: tjwang@partners.org

Contact: Clary Clish, Broad Institute of MIT and Harvard, Cambridge, Mass.

e-mail: clary@broadinstitute.org

3. Hsiao, G. et al. Am. J. Physiol. Endocrinol. Metab. 300, E164-E174 (2011)

\section{COMPANIES AND INSTITUTIONS MENTIONED}

Broad Institute of MIT and Harvard, Cambridge, Mass. Duke University School of Medicine, Durham, N.C. GlaxoSmithKline plc (LSE:GSK; NYSE:GSK), London, U.K. Harvard Medical School, Boston, Mass.

Massachusetts General Hospital, Boston, Mass.

Metabolon Inc., Research Triangle Park, N.C.

Takeda Pharmaceutical Co. Ltd. (Tokyo:4502), Osaka, Japan Tethys Bioscience Inc., Emeryville, Calif. 